\title{
Microencapsulation of Live Cells in Synthetic Polymer Capsules
}

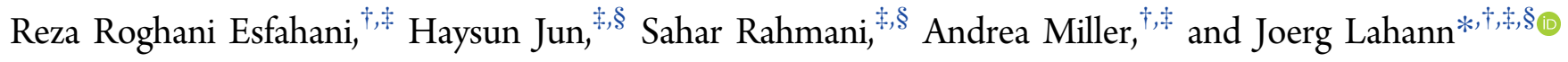 \\ ${ }^{\dagger}$ Chemical Engineering Department, ${ }^{\star}$ Biointerface Institute, and ${ }^{\S}$ Biomedical Engineering Department, University of Michigan, Ann \\ Arbor, Michigan 48109, United States
}

\section{Supporting Information}

ABSTRACT: In cell therapies, it is advantageous to encapsulate live cells in protective, semipermeable microparticles for controlled release of cytokines, growth factors, monoclonal antibodies, or insulin. Here, a modified electrospraying approach with an organic solution of poly(lactide-co-glycolide) (PLGA) polymer is used to create synthetic PLGA capsules that effectively protect live cells. Using a design of experiment (DOE) methodology, the effect of governing jetting parameters on encapsulation efficiency, yield, and size is systematically evaluated. On the basis of this analysis, the interaction between bovine serum albumin concentration and core flow rate is the most dominant factor determining core encapsulation efficiency as well as the microcapsule size. However, the interaction between shell solvent ratio and shell flow rate predominantly defines the particle yield. To

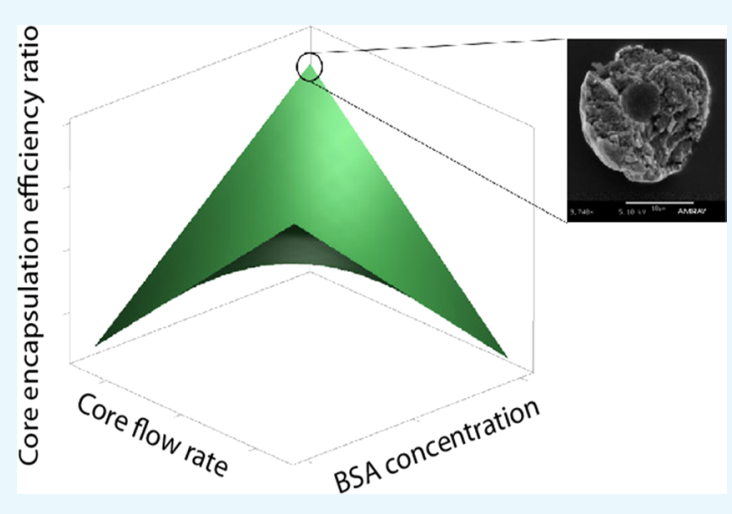
validate these findings, live cells have been successfully encapsulated in microcapsules using optimized parameters from the DOE analysis and have survived the electrohydrodynamic jetting process. Extending the currently available toolkit for cell microencapsulation, these biodegradable, semi-impermeable cell-laden microcapsules may find a range of applications in areas such as tissue engineering, regenerative medicine, and drug delivery.

\section{INTRODUCTION}

In recent years, cell-based therapies have emerged as promising strategies intended to augment conventional drug-based therapies. In particular, cell therapies have been developed for local production of a range of biologics such as cytokines, growth factors, monoclonal antibodies, and insulin. ${ }^{1,2}$ However, the direct administration of foreign cells using intradermal techniques has been plagued by immunorejection of the transplanted cells. Alternatively, encapsulation of live cells in microparticles has been evaluated as a potential method to mitigate unwanted host responses. ${ }^{3}$ Microcapsules can provide a protective environment for cells to proliferate and generate therapeutic agents, while permitting bidirectional diffusion of oxygen, nutrients, and metabolites. ${ }^{4,5}$ So far, natural hydrogels have been the material of choice for microencapsulation of cells because their processing is done under physiologically compatible conditions. ${ }^{6}$ In particular, agarose, alginate, and collagen are widely used natural hydrogels for cellular encapsulation. ${ }^{7}$ Although the permeable nature of these hydrogels permits cell proliferation and rapid diffusion, the particle design space is potentially restricted by factors such as temperature, $\mathrm{pH}$, or incorporation of cross-linking agents needed to crosslink the natural hydrogels. ${ }^{8,9}$ In principle, synthetic polymers provide a wider experimental spectrum that allows for systematic changes in the particle size, porosity, and membrane thickness. However, a major challenge has been to avoid exposure of cells to organic solvents and nonphysiological temperatures needed for the processing of synthetic polymers. A potential solution offers the use of a core-shell structure, wherein the cells are encapsulated in the core and are fully separated from the polymer shell throughout the fabrication process.

The structure of core-shell particles creates a biologically safe environment for the cell in the core, while utilizing polyesters such as poly(lactide-co-glycolide) (PLGA) in the shell creates a protective layer that allows for the study of individual cells and provides the means to deliver the cells into various environments such as low/high $\mathrm{pH}$ conditions and high enzyme tissues. It also allows individual cell entrapment, which has important applications in single cell-based biology, development of biosensor circuits, and bioreactors. It also protects the cells against lethal stressors resembling the sporulation process in nature. ${ }^{10,11}$ Moreover, because the viability of the cells inside the particles depends on the permeability of the particles, individual cell encapsulation eliminates the problem of cells farther from the barrier receiving less nutrients.

Encapsulation of cells in microparticles has been achieved using several different techniques, such as electrostatic spraying, emulsion, micro-nozzle array, interfacial polymerization, and extrusion methods. ${ }^{12-16}$ Using a modified electrospraying approach, we now report a new type of polymer capsule that is composed of PLGA shells protecting live cells inside the core.

Received: May 8, 2017

Accepted: June 7, 2017

Published: June 21, 2017 


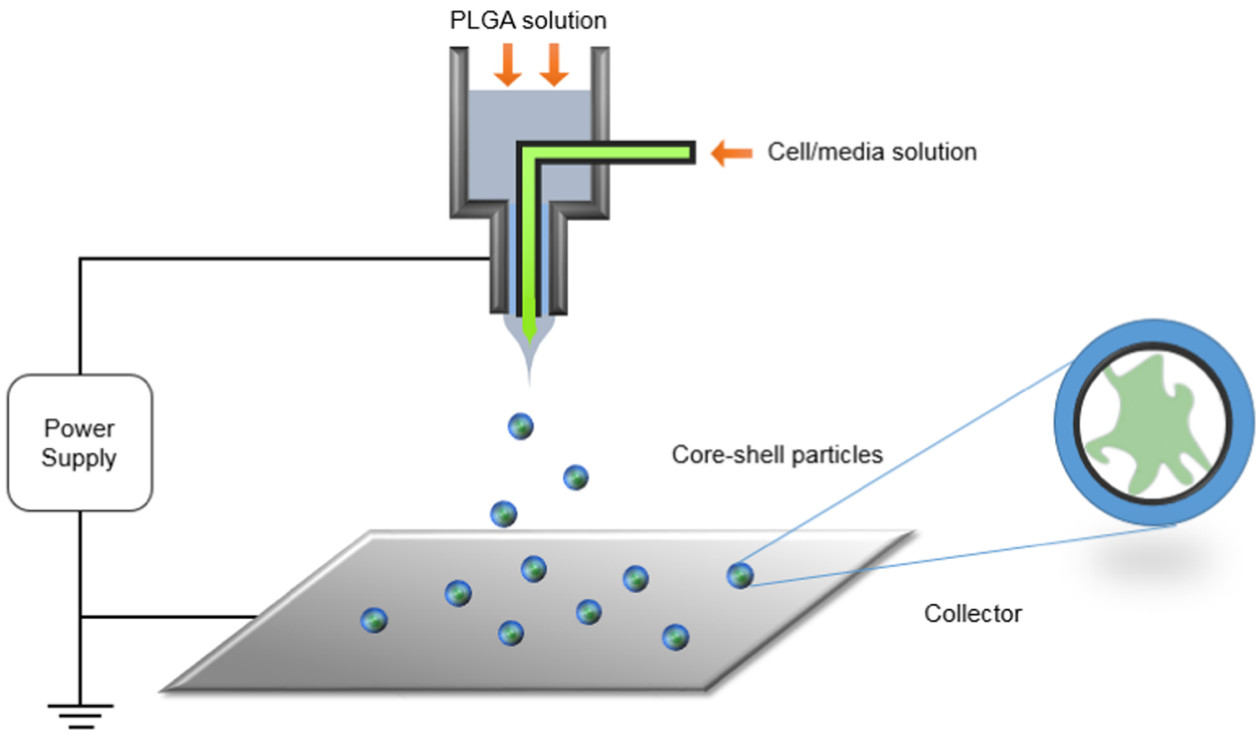

Figure 1. Schematic illustration of coaxial electrohydrodynamic (EHD) jetting. The viscoelastic polymer shell and the cell-loaded core solutions are forced through a coaxial needle under applied electric potential resulting in cell encapsulated core-shell microparticles.

\section{RESULTS AND DISCUSSION}

To prepare polymer capsules containing live cells, the PLGA solution and a cell-loaded core solution are simultaneously driven through a needle using a sufficiently high electrical voltage. This work has been inspired by past studies that report significant control over critical properties of multicompartmental nano- and microparticles. ${ }^{17-20}$ As previously reported, the viscoelastic polymeric solution at the tip of the needle forms into a droplet and becomes stable under an applied electric potential. This stability is the result of the equilibrium between the applied electric force and the surface tension in the viscoelastic droplet. ${ }^{21}$ Rapid evaporation of solvents from both the shell and the core results in the fabrication of core-shell microparticles. Typically, particles fabricated using this technique feature small size distribution with high surface to volume ratios that enhance the transfer of oxygen and nutrients. ${ }^{22}$ The schematic of this process is depicted in Figure 1.

Control over encapsulation efficiency, yield, and size of the particles are of significant importance for cell-based therapies. Importantly, this study utilizes a design of experiment (DOE) methodology to identify optimum parameters for microencapsulation of living cells in biodegradable polymer capsules. DOE methodology not only predicts the main factors affecting the encapsulation efficiency, yield, and size of the particles, but also determines the important interaction factors. Factorial DOE was utilized in this study to investigate the contribution of each input parameter on the process outputs. An improved insight into the relevance of the applicable parameters along with their interactions on the output parameters such as efficiency, size, and yield can enhance our understanding of the particle fabrication process.

The DOE methodology offers an efficient approach in the determination of significant contributing factors while reducing bias and the total number of trials. In electrospraying, there are many controllable parameters that have an impact in particle fabrication, which include surface tension, dielectric constant, density, viscosity of the solution, and the vapor pressure of solvent. $^{23,24}$ These parameters are all dependent on the composition of the jetting solution. Secondary factors such as applied voltage, needle tip to collector distance, and environmental factors like ambient temperature and humidity also affect the experimental outcome. However, in a typical experiment, these secondary factors remain approximately constant. We note that the applied voltage required to deform the coaxial meniscus into the corresponding Taylor cone may vary slightly between experiments. ${ }^{25}$ A set of 16 experimental trials was conducted through the study of the experimental space as described in the design matrix (Table 1). The order of

Table 1. Factors with the Corresponding Levels Used in the Construction of the Experimental Space

\begin{tabular}{|c|c|c|c|}
\hline \multirow[b]{2}{*}{$\begin{array}{l}\text { experimental } \\
\text { factors }\end{array}$} & \multirow[b]{2}{*}{$\begin{array}{c}\text { number of } \\
\text { levels }\end{array}$} & \multicolumn{2}{|c|}{ model parameters' factor levels studied } \\
\hline & & low & high \\
\hline $\begin{array}{l}\text { BSA } \\
\text { concentration } \\
\text { (A) }\end{array}$ & 2 & $10 \% \mathrm{w} / \mathrm{v}$ & $20 \% \mathrm{w} / \mathrm{v}$ \\
\hline $\begin{array}{l}\text { core flow rate } \\
\text { (B) }\end{array}$ & 2 & $0.02 \mathrm{~mL} \mathrm{~h}^{-1}$ & $0.1 \mathrm{~mL} \mathrm{~h}^{-1}$ \\
\hline $\begin{array}{l}\text { PLGA } \\
\text { concentration } \\
\text { (C) }\end{array}$ & 2 & $10 \% \mathrm{w} / \mathrm{v}$ & $15 \% \mathrm{w} / \mathrm{v}$ \\
\hline $\begin{array}{l}\text { shell solvent ratio } \\
\text { (D) }\end{array}$ & 2 & $90: 10 \mathrm{v} / \mathrm{v} \mathrm{CHCl}_{3} / \mathrm{DMF}$ & $\begin{array}{l}97: 3 \mathrm{v} / \mathrm{v} \\
\mathrm{CHCl}_{3} / \mathrm{DMF}\end{array}$ \\
\hline $\begin{array}{l}\text { shell flow rate } \\
\text { (E) }\end{array}$ & 2 & $0.1 \mathrm{~mL} \mathrm{~h}^{-1}$ & $0.5 \mathrm{~mL} \mathrm{~h}^{-1}$ \\
\hline
\end{tabular}

the experiments was randomized to reduce bias. ${ }^{26}$ Table 1 lists the five experimental factors that were chosen at two levels for this study. By definition, levels are defined as the specific quantities assumed for each of the factors. These five experimental factors were bovine serum albumin (BSA) concentration (A), core flow rate (B), PLGA concentration (C), shell solvent ratio (D), and shell flow rate (E). It was assumed based on prior experience that these factors have the most effect on the morphology and yield of core-shell particles. The specific quantities selected for each parameter were based on initial studies and the stability of the Taylor cone during the EHD jetting process. Preliminary data (not shown) suggested that the stability of the Taylor cone is predominantly 
(a)

A

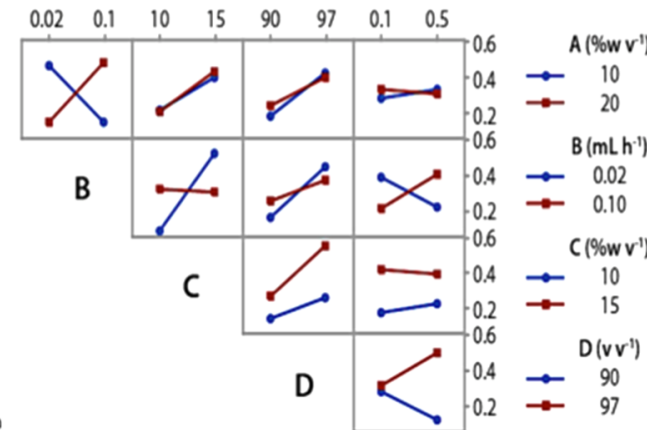

(b)

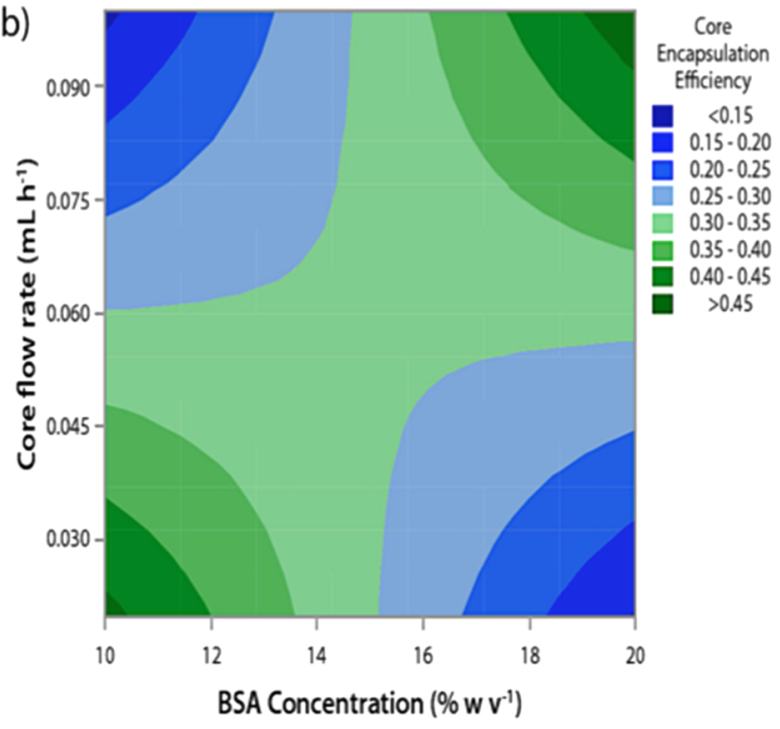

Figure 2. Determination of significant contributing factors in encapsulation efficiency. (a) Interaction plot for core encapsulation efficiency. (b) Contour plot of core encapsulation efficiency vs. core flow rate and BSA concentration, where PLGA concentration, shell solvent ratio, and shell flow rate are all held constant at $12.5 \% \mathrm{w} / \mathrm{v}, 93.5: 6.5 \mathrm{v} / \mathrm{v} \mathrm{CHCl} 3 / \mathrm{DMF}$, and $0.3 \mathrm{~mL} \mathrm{~h}^{-1}$, respectively. (c) Pareto chart of significant contributing factors in core encapsulation efficiency. Experimental factors are as follows: (A) BSA concentration, (B) core flow rate, (C) PLGA concentration, (D) shell solvent ratio, and (E) shell flow rate.

dependent on the polymer solutions used for jetting. These confining parameters include the volatility, concentration, and the dielectric constant of the solutions used for the electrospraying process. The more concentrated the polymer solution is, the more gradual the precipitation process becomes, which results in fabrication of particles with higher sphericity. ${ }^{27}$ Here, PLGA solutions with a mixture of chloroform and dimethylformamide (DMF) with polymer concentrations of $10 \%(\mathrm{w} / \mathrm{v})$ and $15 \%(\mathrm{w} / \mathrm{v})$ were chosen because higher PLGA concentrations resulted in fiber formation. Chloroform has a higher volatility than DMF and constitutes the majority of the solvent. On the other hand, DMF has a higher dielectric constant and tends to increase the stability of the Taylor cone during particle fabrication. Consequently, chloroform/DMF ratios of 97:3 and 90:10 (v/v) were chosen as the two levels for this study. The addition of BSA to the core solution was thought to improve the overall biocompatibility of polymer microcapsules and to increase cell viability. BSA also increased the viscosity of the core solution and thus stabilized the EHD jet. The two levels for the BSA concentration were $10 \%(\mathrm{w} / \mathrm{v})$ and $20 \%(\mathrm{w} / \mathrm{v})$. Flow rates of 0.1 and $0.5 \mathrm{~mL} \mathrm{~h}^{-1}$ were selected for the shell solution, and 0.02 and $0.1 \mathrm{~mL} \mathrm{~h}^{-1}$ were chosen for the core solution, by taking into account the diameter differences in the jetting needles and to reduce the polydispersity of the particles that are often observed with higher flow rates. ${ }^{28,29}$

Once the design space for each parameter was determined, particles were prepared under conditions defined by the overall matrix of all available options. After completion of the 16 experimental runs, the data was analyzed to identify the effects of each contributing factor in the fabrication of core-shell particles. The DOE analysis was carried out for three different experimental responses: (i) core encapsulation efficiency, (ii) yield, and (iii) size.

2.1. Core Encapsulation Efficiency. Core encapsulation efficiency is the fraction of particles that feature a core/shell architecture, that is, the fraction of particles that are intact microcapsules. Because both the core (red) and the shell (blue) compartments were fluorescent, the microcapsules could be analyzed using flow cytometry. Excitation intensity of DAPI (blue fluorescence) from a dye added to the polymer shell and Texas red (red fluorescence) from a dye added to the core revealed the percentage of particles in each experiment that were fully intact microcapsules. After the collection of data for each of the 16 experimental runs, DOE analysis was performed. Apart from studying the impact of the five main effects, DOE can also assist in the identification of statistically significant two-factor interactions. Subsequently, the interaction effects that were least significant (highest $p$-value) in core encapsulation efficiency were systematically eliminated from the model until each interaction had a $p$-value below 0.05 (95\% confidence level) (Figure 2).

As shown in Figure 2, four two-factor interactions and two main factors are significantly influencing the core encapsulation efficiency at a 95\% confidence level. The Pareto chart of effects (Figure 2C) reveals the significance of each effect against a reference line. Any main or two-factor interaction effect that surpasses the reference line is considered statistically significant $(p$-value $<0.05)$

For core encapsulation efficiency, the two main factors are the PLGA concentration and the shell solvent ratio. Relevant two-factor interactions include $\mathrm{AB}$ (BSA concentration and core flow rate), $\mathrm{BC}$ (core flow rate and PLGA concentration), $\mathrm{BE}$ (core flow rate and shell flow rate) and DE (shell solvent ratio and shell flow rate). Interestingly, the most dominant twofactor interaction is $\mathrm{AB}$, both of which are independent of the shell flow. This dominance is apparent through the interaction plot, wherein $A B$ has the largest slope difference (Figure 2C). In an interaction plot, the greater the difference in slopes between the two lines, the greater the degree of interaction between those factors. Hence, parallel lines in the interaction plot indicate the absence of interactions. Knowledge of the 

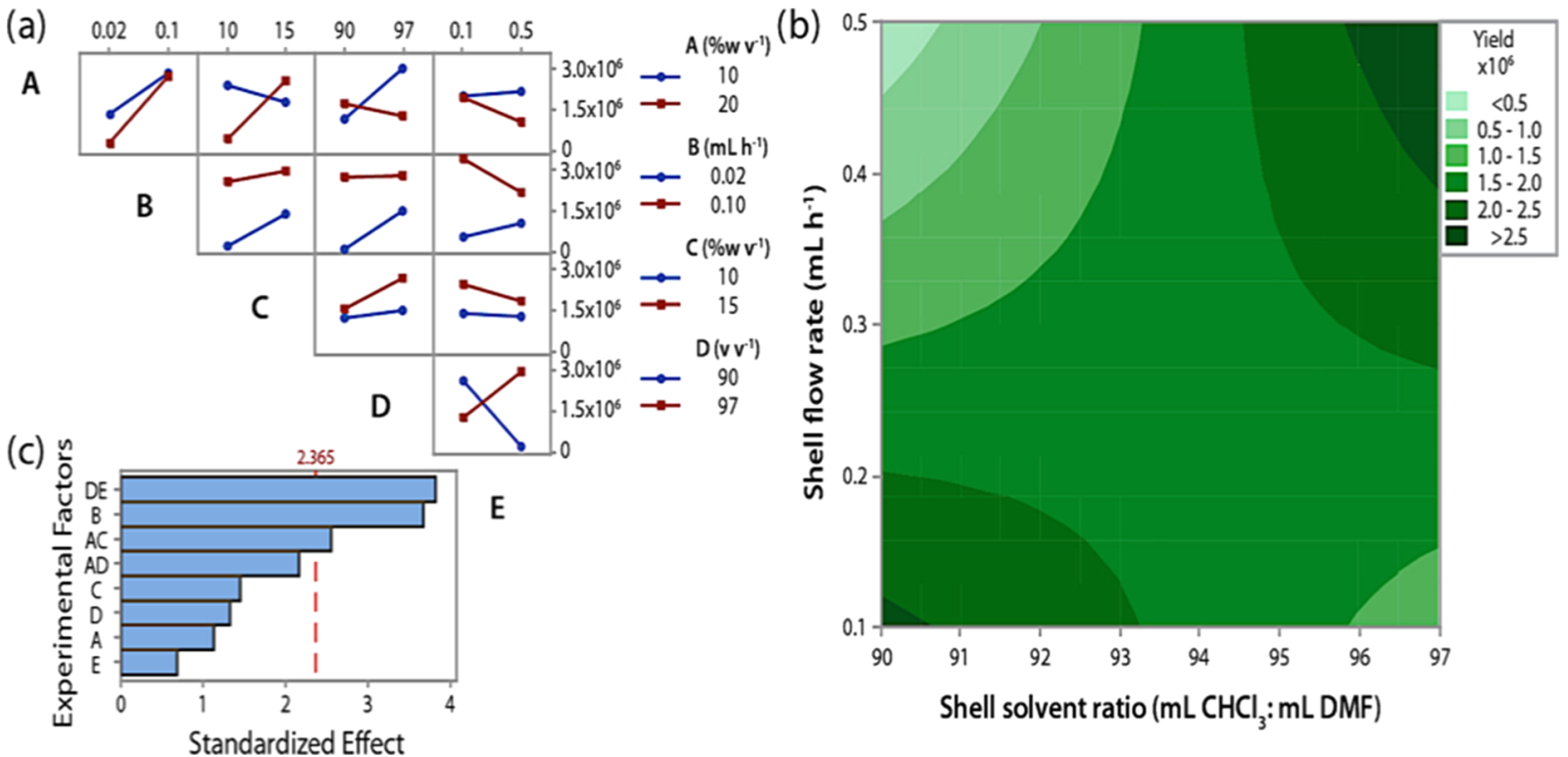

Figure 3. Determination of significant contributing factors in microcapsule yield. (a) Interaction plot for the yield of microparticles. (b) Contour plot of yield vs shell flow rate and shell solvent ratio, where BSA concentration, core flow rate, and PLGA concentration are all held constant at $15 \% \mathrm{w} / \mathrm{v}$, $0.06 \mathrm{~mL} \mathrm{~h}^{-1}$, and $12.5 \% \mathrm{w} / \mathrm{v}$, respectively. (c) Pareto chart of significant contributing factors in determination of yield. Experimental factors are as follows: (A) BSA concentration, (B) core flow rate, (C) PLGA concentration, (D) shell solvent ratio, and (E) shell flow rate.
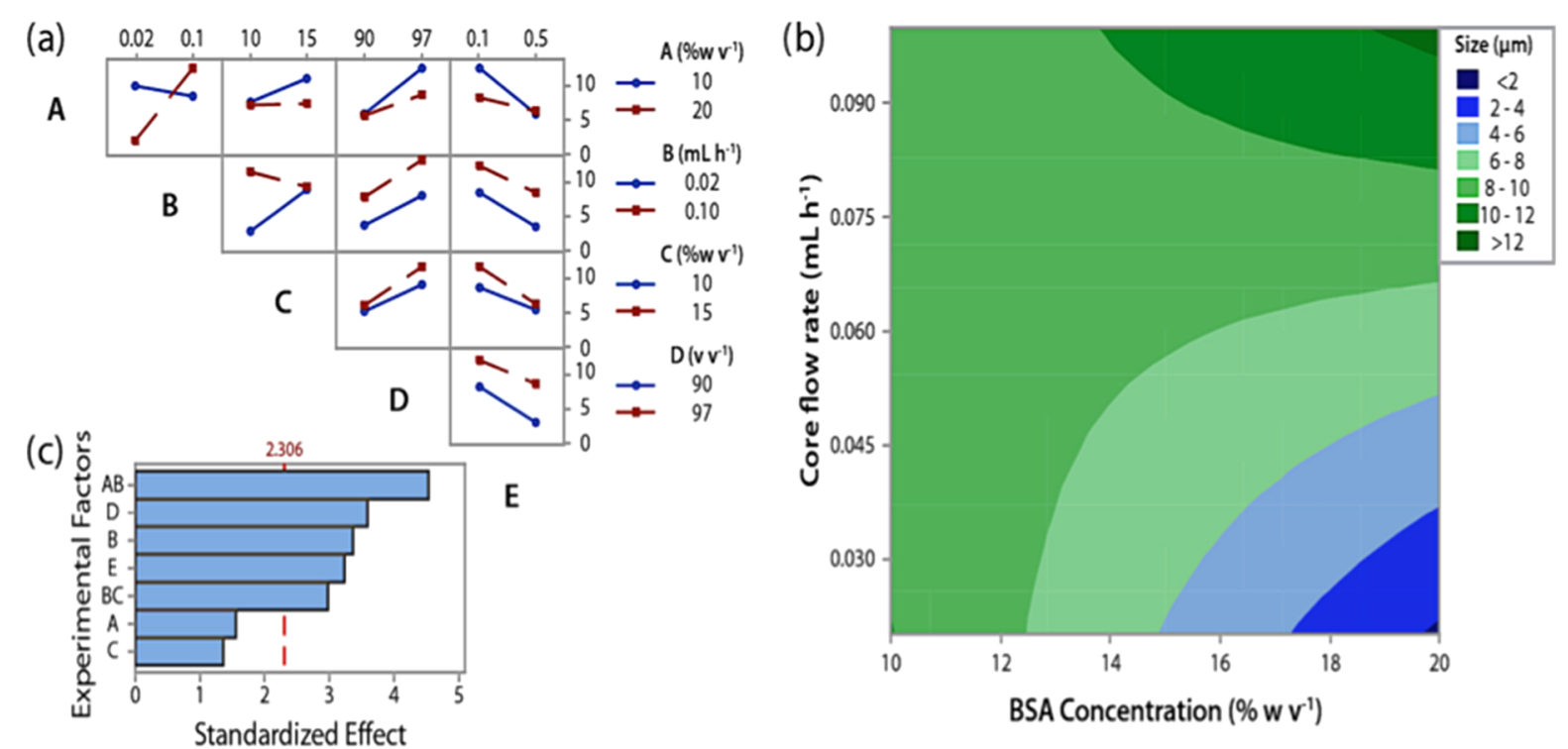

Figure 4. Determination of significant contributing factors in microcapsule size. (a) Interaction plot for the size of microparticles. (b) Contour plot of size vs core flow rate and BSA concentration, where PLGA concentration, shell solvent ratio, and shell flow rate are all held constant at $12.5 \% \mathrm{w} / \mathrm{v}$, 93.5:6.5 $\mathrm{CHCl}_{3} / \mathrm{DMF}$, and $0.3 \mathrm{~mL} \mathrm{~h}^{-1}$, respectively. (c) Pareto chart of significant contributing factors in the determination of size. Experimental factors are as follows: (A) BSA concentration, (B) core flow rate, (C) PLGA concentration, (D) shell solvent ratio, and (E) shell flow rate.

main and two-factor interactions that contribute to the encapsulation efficiency along with their absolute value was utilized to generate a model (Figure S1). This predictive model, which is not shown here, was then used to generate a contour plot. In Figure 2B, the relationship between core encapsulation efficiency, core flow rate, and BSA concentration is summarized in the contour plot: Core flow rate and BSA concentration have to be selected concurrently to have a positive effect on the efficiency. The range of these parameters in the lower left corner is roughly $0.02-0.035 \mathrm{~mL} \mathrm{~h}^{-1}$ for the core flow rate and $10-12 \%(\mathrm{w} / \mathrm{v})$ for the BSA concentration. In the upper right hand corner, this range is between 0.09 and $0.1 \mathrm{~mL} \mathrm{~h}^{-1}$ for the core flow rate and between 19 and $20 \%(\mathrm{w} / \mathrm{v})$ for the BSA concentration.

2.2. Microcapsule Yield. Here, the microcapsule yield is defined as the number of particles prepared per mass of PLGA used. The number of particles was measured using a hemocytometer. Figure 3 displays the analyses that followed the experimental approach described in the previous section.

Similar to core encapsulation efficiency, systematic elimination of insignificant parameters from the model yielded a Pareto chart that identified the main factors and two-factor interactions.

As shown in Figure 3C, the main contributing factor is core flow rate. Moreover, there are two significant two-factor 


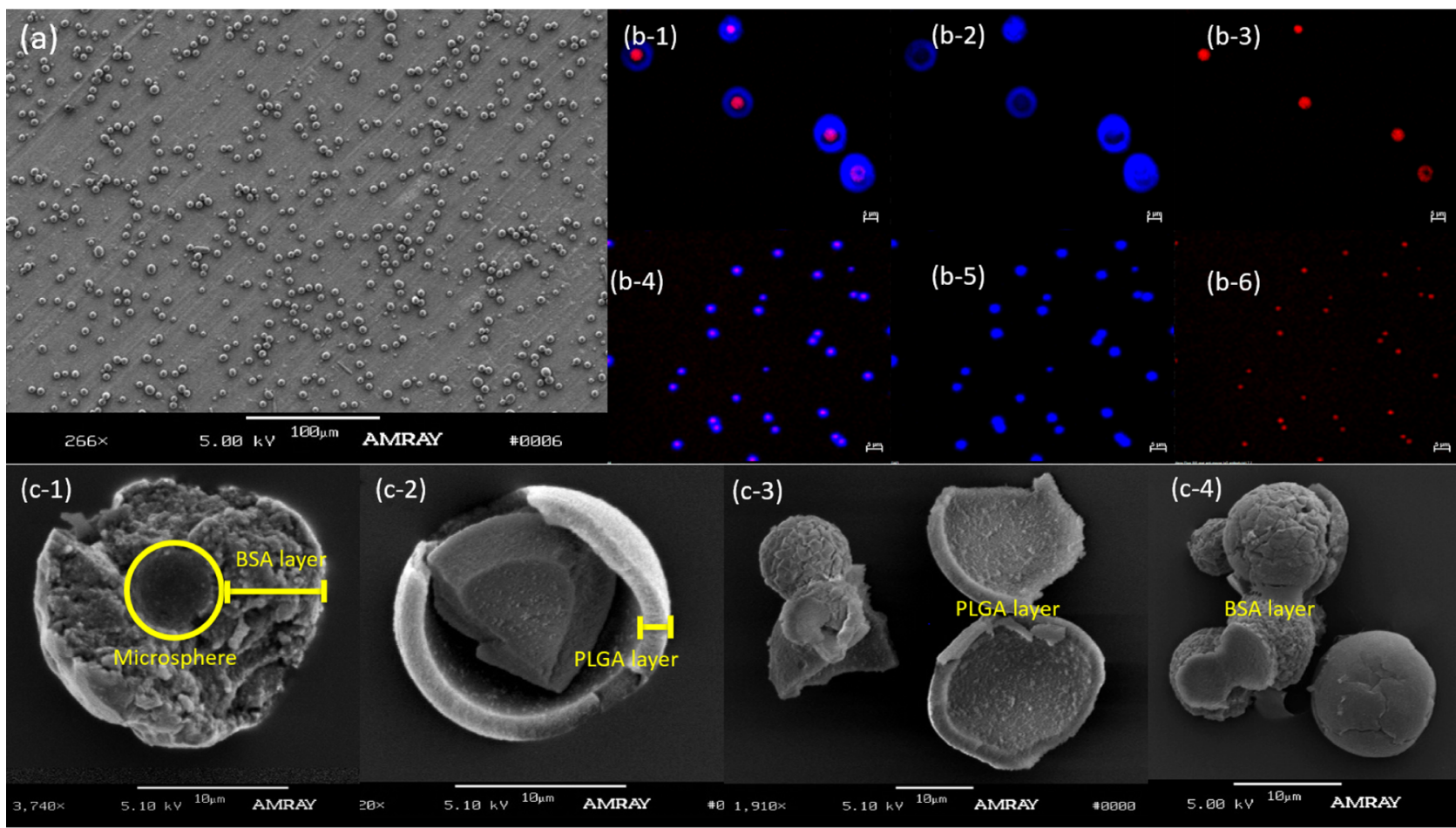

Figure 5. Fabrication of core-shell microparticles: (a) SEM image of uniform microparticles, (b-1) superimposed DAPI and Texas red channels of CLSM image of microparticles; (b-2) CLSM image of the DAPI channel, showing the PLGA shell layer containing blue dye; (b-3) CLSM image of the Texas red channel, showing the BSA core layer containing red silica microspheres; (b-4) lower-resolution superimposed CLSM image of microparticles; (b-5) zoomed-out CLSM image of the DAPI channel, showing the PLGA shell layer containing blue dye; (b-6) zoomed-out CLSM image of the Texas red channel, showing the BSA core layer containing red silica microspheres; (c-1) SEM image of cross-sectioned BSA layer containing a silica microsphere; (c-2) SEM image of cross-sectioned PLGA layer; (c-3) SEM image of cross-sectioned PLGA layer; and (c-4) SEM image of cross-sectioned BSA layer.

interactions: DE (shell solvent ratio and shell flow rate) and AC (BSA concentration and PLGA concentration). As described in the case of core encapsulation efficiency, the slope differences in the interaction plot are indicative of the significance of each interaction (Figure 3A). Because DE (shell solvent ratio, shell flow rate) has the largest slope differences and utmost significance, a contour plot displaying the relationship between yield, shell flow rate, and shell solvent ratio has been prepared (Figure 3B). Similar to core encapsulation efficiency analysis, the contour plot was generated using a predictive model, which is not shown here (Figure S2). According to Figure 3B, to maximize yield, a shell flow rate in the range of $0.4-0.5 \mathrm{~mL} \mathrm{~h}^{-1}$ is recommended; however, the shell/solvent ratio should be in the range of 96:4-97:3 (v/v) chloroform and DMF. Note that even though $\mathrm{AD}$ (BSA concentration and shell solvent ratio) did not have a significant effect on the yield of core-shell particles, it was not eliminated in the analysis process because the predictive model used in development of the contour plot had a significantly higher coefficient of determination $\left(R^{2}\right)$, when including this two-factor interaction in the analysis.

2.3. Microcapsule Size. In addition to core encapsulation efficiency and yield, particle size is a critical property for any biomedical application involving cell-laden microcapsules. ${ }^{30} \mathrm{~A}$ corresponding DOE analysis was thus also carried out to determine the significant main and interaction factors affecting the size of core-shell particles. The data for this analysis are presented in Figure 4.

According to the size distribution Pareto chart (Figure 4C), there are three main factors and two two-factor interactions that significantly influence the size of microcapsules. The three main factors are the shell solvent ratio, the core flow rate, and the shell flow rate. The two-factor interactions are $A B$ (BSA concentration, core flow rate) and $\mathrm{BC}$ (core flow rate, PLGA concentration). Because the interaction between BSA concentration and core flow rate is the most dominant, a contour plot displaying the relationship between size, core flow rate, and BSA concentration was generated (Figure 4B). Similar to core encapsulation efficiency and yield analysis, the contour plot was generated using a predictive model, which is not shown here (Figure S3). According to the contour plot, the size of the microcapsules is independent of the core flow rate for BSA concentrations between 10 and $12.5 \%(\mathrm{w} / \mathrm{v})$. These BSA concentrations also yielded the most monodisperse particles. However, as the concentration of BSA increases, the variance of the particle size increases concurrently. Because the goal of the experiment is to encapsulate primary mammalian cells with an estimated diameter of $8-10 \mu \mathrm{m} ; 8-10 \mu \mathrm{m}$ was defined as the target value for the microcapsules. On the basis of this analysis, we came to know that there are two distinct regions that result in the highest encapsulation efficiency. Those regions were with core flow rates between 0.02 and $0.035 \mathrm{~mL} \mathrm{~h}^{-1}$ in conjunction with $10-12 \%(\mathrm{w} / \mathrm{v})$ BSA concentrations, or core flow rates between 0.09 and $0.1 \mathrm{~mL} \mathrm{~h}^{-1}$, if the BSA concentrations were between 19 and $20 \%(\mathrm{w} / \mathrm{v})$. Because both encapsulation efficiency and size are dependent on the combination of core flow rate and BSA concentration, an overlapping region with core flow rate $0.02-0.035 \mathrm{~mL} \mathrm{~h}^{-1}$ and $10-12 \% \mathrm{w} / \mathrm{v}$ BSA concentration can be identified, wherein the encapsulation efficiency is maximum and the size target can be met. 


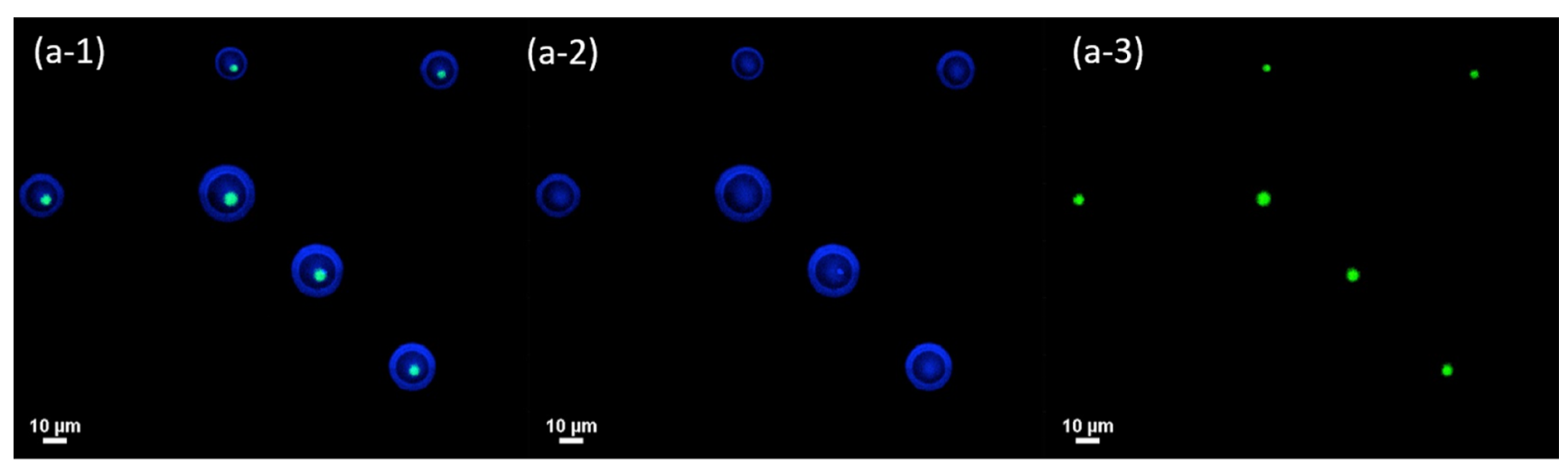

(b-1) Microparticles without GFP-NIH3T3 cells (control)

(b-2) Microparticles encapsulating GFP-NIH3T3 cells
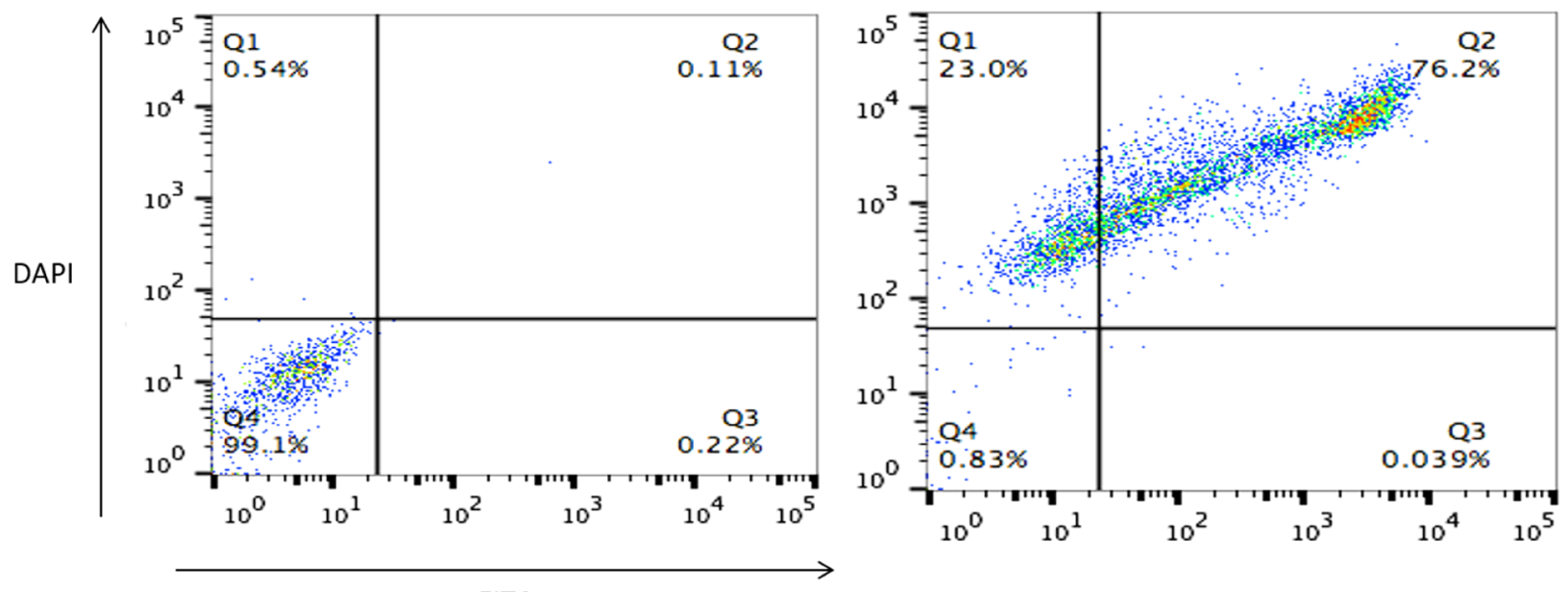

FITC

Figure 6. GFP-NIH3T3 cell microencapsulation. (a-1) CLSM image of GFP-NIH3T3 cell-core/PLGA-shell microparticles (superimposed green and blue channels); (a-2) CLSM image of PLGA shells (blue channel); (a-3) CLSM image of GFP-NIH3T3 cells in BSA core (green channel); (b1) flow cytometry analysis of encapsulation efficiency of core-shell microparticles not encapsulating GFP-NIH3T3 cells with PLGA (blue dye) shell and BSA core layers (control); and (b-2) flow cytometry analysis of encapsulation efficiency of core-shell microparticles encapsulating GFPNIH3T3 cells with PLGA (blue dye) shell and BSA core layers.

2.4. Preparation of Cell-Laden Microcapsules. The relationships demonstrated in Figures 2-4 provided a detailed understanding of the main operating parameters for the fabrication of uniform, cell-laden microcapsules in high yields. On the basis of this analysis, a BSA concentration of $10 \% \mathrm{w} / \mathrm{v}$, core flow rate of $0.02 \mathrm{~mL} \mathrm{~h}^{-1}$, PLGA concentration of $10 \% \mathrm{w} /$ $\mathrm{v}$, shell solvent ratio of 97:3 v/v chloroform/DMF, and shell flow rate of $0.5 \mathrm{~mL} \mathrm{~h}^{-1}$ were selected as the operating parameters for cell encapsulation. Core-shell particles were then fabricated using these parameters to validate the statistical model. Because this experiment was carried out to evaluate the practicality and the feasibility of core-shell particle fabrication, cells were not added to the core solution. Instead, red silica microspheres were added to the core solution to be encapsulated instead of cells. The fabricated core-shell particles were then collected and suspended in an optimum cutting temperature gel. The gel was frozen at $-70{ }^{\circ} \mathrm{C}$ and sectioned via a cryosectioning machine. These sectioned particles were mounted on a glass slide and imaged via scanning electron microscopy (SEM) and confocal laser scanning microscopy (CLSM). These images were used to qualitatively evaluate the outcome of this experiment. Figure 5 presents the images obtained from EHD jetting of core-shell particles and their internal architecture.
As evident from the images displayed in Figure 5, core-shell particles were successfully fabricated using the parameters derived from the aforementioned analysis. From the SEM image, it can be deduced that the microparticles have been fabricated in high yields (Figure 5A). The CLSM images along with cross-sectional SEM images reveal the success of coreshell particle formation and their morphology. In CLSM images, red silica microspheres and blue dye were used as the markers for the core and shell, respectively (Figure 5B). Figure 5C presents the SEM images of the sectioned particles after cryosectioning. It is evident that the silica microspheres have been successfully encapsulated within the BSA layer to form the core, and the core has been entrapped within the PLGA shell.

2.5. Cell Viability. After ensuring the synthesis of coreshell particles, microencapsulation of live NIH3T3 fibroblast cells was investigated. The core and shell solutions were prepared as explained in detail in the Section 4 and fabricated using the parameters obtained from the DOE analysis. The polymeric shell solution contained a blue dye, and NIH3T3 cells were stained with the live/dead assay. The particles were jetted into a solution of Dulbecco's modified Eagle's medium (DMEM) to ensure that the cells would survive. Figure 6 presents the data obtained through this analysis.

Figure $6 \mathrm{~A}$ presents the confocal images of the cell-loaded core-shell particles. An examination of the fluorescent signals 


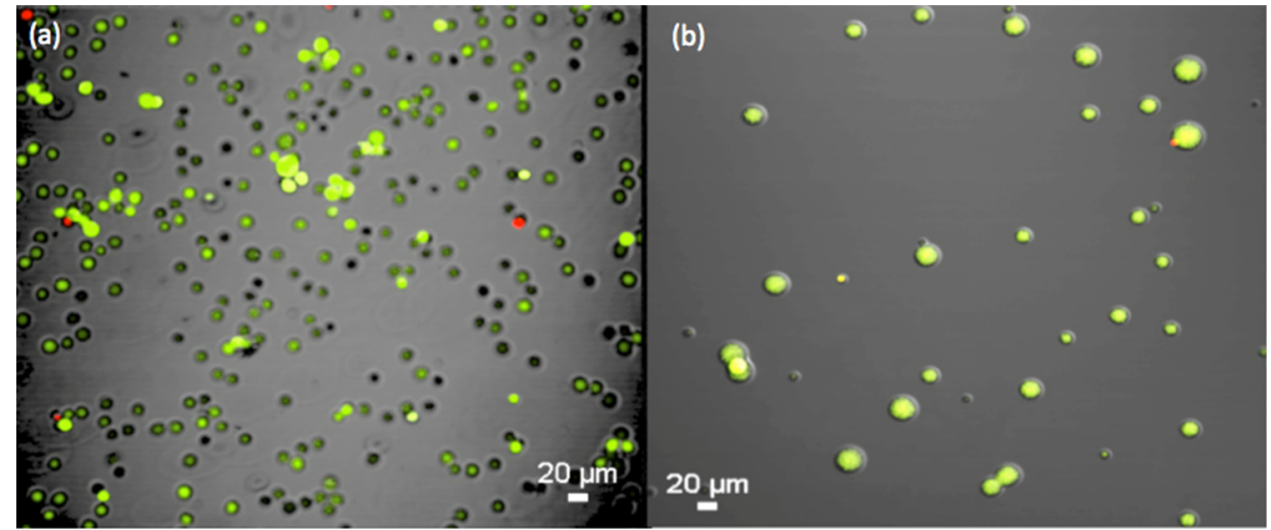

Figure 7. In vitro cell viability of NIH3T3 cells encapsulated in core-shell microparticles. (a) CLSM image of NIH3T3 cells stained with Live/Dead assay kit (green: live/red: dead) demonstrating NIH3T3 cells before encapsulation in core-shell particles. (b) CLSM image of NIH3T3 cells stained with Live/Dead assay kit (green: live/red: dead) encapsulated in core-shell microparticles.

of blue and green dyes using flow cytometry reveals that $76.2 \%$ of the cells were alive and had been successfully encapsulated in the core. Figure $6 \mathrm{~B}$ shows the dot plot of the particles wherein the control microparticles, negative for the green signal, fell in the lower left quadrant. Microparticles loaded with stained cells were confined in the upper two quadrants characterized by their fluorescence signal. This evaluation is also proof of the viability of cells after encapsulation and shows that the cells were capable of withstanding the applied voltage. Figure 7 shows the confocal images obtained before and after microencapsulation of stained cells. Figure 7A shows the stained NIH3T3 cells before entrapment in microcapsules. The color green represents live cells and red represents the dead ones. Figure 7B demonstrates the stained NIH3T3 cells after microencapsulation in PLGA core-shell particles.

\section{CONCLUSIONS}

In this study, a modified electrospraying approach was successfully utilized to fabricate core-shell particles encapsulating live cells. The effect of each jetting parameter on encapsulation efficiency, yield, and size was studied systematically using DOE methodology. Contour plots of the relationship between each response and the most dominant affecting experimental factors were displayed. It was demonstrated that the interactions between BSA concentration and core flow rate is the most dominant factor affecting core encapsulation efficiency and size of the core-shell particles. Furthermore, it was shown that the interaction between shell solvent ratio and shell flow rate is the most dominant factor affecting the yield of core-shell particles. We further identified the optimum operating parameters for core-shell particles synthesis: $10 \%$ $\mathrm{w} / \mathrm{v}$ BSA concentration, $0.02 \mathrm{~mL} \mathrm{~h}^{-1}$ core flow rate, $10 \% \mathrm{w} / \mathrm{v}$ PLGA concentration, 97:3 chloroform/DMF v/v solvent ratio, and $0.5 \mathrm{~mL} \mathrm{~h}^{-1}$ shell flow rate. For proof of concept, NIH3T3 cells were successfully encapsulated in core-shell microparticles using the parameters obtained from the DOE analysis. It was shown that the cells were alive and able to withstand the applied electric potential during EHD jetting. In the future, cell delivery via microencapsulation of cells in biodegradable, semiimpermeable particles may have applications in tissue engineering, regenerative medicine, as well as vehicles for the production and secretion of hormones and growth factors, such as insulin.

\section{EXPERIMENTAL SECTION}

4.1. Materials. Poly(lactic-co-glycolic acid) (PLGA, 50:50 lactic acid to glycolic acid ratio, and molecular weight $\left(M_{\mathrm{w}}\right)$ of $44 \mathrm{kDa}$ ) was purchased from Corbin (Lenexa, KS). BSA, chloroform, DMF, Tween 20, and poly[( $m$-phenylenevinylene)-alt-(2,5-dihexyloxy-p-phenylenevinylene)] (MEHPV) were obtained from Sigma Aldrich (St. Louis, MO) and used as received. $\mathrm{m}$-PEG-rhodamine $(5 \mathrm{kDa})$ was used as received from Creative PEGWorks (Chapel Hill, NC), and the OCT compound was purchased from Fisher HealthCare (Houston, TX). The mouse embryonic fibroblast cell line, NIH3T3 cells were obtained from ATCC (Manassas, VA). Calcein AM and ethidium homodimer were both purchased from Life Technologies (Carlsbad, CA). DMEM and tryspin were obtained from Gibco (Grand Island, NY).

4.2. Preparation of Jetting Solutions. PLGA was dissolved in a solvent consisting of chloroform and DMF to be used as the shell. The formulation of this polymeric solution was varied in accordance with the DOE matrix outlined in Section 2.4 (Table 1). BSA was dissolved in the water/ rhodamine mixture, and the resulting solvent was used as the core solution, with its formulation varied in accordance with the DOE matrix (Table 1) to optimize the particle formulation. After the particle formulation was optimized, the core solution was replaced with NIH3T3 mouse fibroblasts suspended in a $10 \%$ Matrigel (Corning, NY) solution at a concentration of 2 million cells $/ \mathrm{mL}$. These NIH3T3 mouse fibroblasts were cultured under standard conditions at $37{ }^{\circ} \mathrm{C}$ with $5 \% \mathrm{CO}_{2}$.

The Matrigel was diluted in DMEM $(4.5 \mathrm{~g} / \mathrm{mL})$ containing 4 $\mathrm{mM}$ L-glutamine, $10 \%$ fetal bovine serum, $1 \%$ penicillinstreptomycin, and 1\% MEM nonessential amino acids (Gibco, Grand Island, NY). The samples were washed with PBS and resuspended in a solution of DMEM and 10\% Matrigel.

4.3. EHD Cojetting. A coaxial needle system, which consisted of a 25-gauge needle centered within an 18-gauge needle, was used to synthesize the core-shell microparticles. The PLGA solution was loaded into the outer needle to make the shell of the particles, and the BSA solution was loaded into the central needle to make the particle core. The flow rates for both the PLGA and BSA solutions were varied according to the DOE matrix (Table 1). After the respective solutions were loaded into the needles, a voltage was applied until a stable Taylor cone was formed, as illustrated in Figure 1. A voltage of $10-12 \mathrm{kV}$ was used for all experiments. For the DOE analysis, 
microparticles were deposited onto a metal sheet and placed in vacuum to dry. For cellular encapsulation, the particles were jetted into DMEM. The solution was then passed through a 40 $\mu \mathrm{m}$ filter followed by a $20 \mu \mathrm{m}$ filter and then centrifuged at $3000 \mathrm{rpm}$. The purified particles were then resuspended in DMEM.

4.4. Formulating the Experimental Design for Microencapsulation of Cells. A half-fraction factorial design with five experimental factors and two levels was used to explore the experimental space and obtain optimal operating parameters. Table 1 illustrated the model parameters used in the construction of the experimental space. The experimental factors are the inputs of the design. Core encapsulation efficiency, yield, and size identify the outputs or the response of the experimental design.

This combination of parameters gave rise to the 16 unique experimental combinations used to map the experimental space. The experimental run order was randomized to minimize bias and ensure that the data points were independent. This elimination was done to avoid convolution of the model.

4.5. Characterization of Jetted Microparticles. Microparticles were imaged with a CLSM using a Nikon A-1 spectral microscope and SEM (Amray 1910 FE-SEM) at the University of Michigan's Microscopy and Imaging Laboratory facilities. For the purpose of the DOE analysis, the samples for confocal and SEM imaging were obtained by placing a microscope cover glass and a silicon chip on the collectors' metal sheet during EHD cojetting. In the case of cellular encapsulation, these samples were obtained from the solution after the centrifugation step outlined in Section 4.3. The sample was then mounted in $7 \mu \mathrm{L}$ of prolong gold (Invitrogen, Eugene, OR) before the confocal analysis. In DOE analysis, confocal images were used to ensure the fabrication of the core-shell particles for each combination of parameters according to the DOE matrix outlined in Section 2.4 (Table 1). The SEM images were used to confirm particle formation and to measure the diameter of microparticles. This measurement was done using Image-J software. ${ }^{31-33}$ For cellular encapsulation, confocal images were used to ensure successful cell encapsulation as determined by staining with a live/dead assay kit. To demonstrate the internal core-shell geometry of these particles, SEM images of microparticles sectioned into $30 \mu \mathrm{m}$ sections were used.

Furthermore, for the purposes of the DOE analysis, the yield was determined by collecting the microparticles and dispersing them in a solution of $0.1 \%$ PBS and Tween 20 . These particles were analyzed using a hemocytometer and the actual concentration of particles per mass of PLGA jetted was calculated.

Core-shell microparticles suspended in PBS with $0.1 \%$ Tween 20 were analyzed by flow cytometry at the University of Michigan flow laboratory. The data obtained from flow cytometry was evaluated to determine the core encapsulation efficiency using the percentage of particles that had both red and blue dyes associated with them. In cellular encapsulation, the control group of particles (no cell encapsulated) and particles encapsulating NIH3T3-GFP cells were prepared as stated and analyzed by flow cytometry to determine the cell encapsulation efficiency. For data analysis, the MoFlo Astrios software was used.

4.6. Cell Viability. The cell viability was evaluated by confocal microscopy using calcein AM and ethidium homodimer. First, the cells were passaged at $80 \%$ confluence using $0.25 \%$ tryspin, which was neutralized with a three-fold excess of the complete medium. The cells were then centrifuged at $300 \mathrm{~g}$ for $5 \mathrm{~min}$ at $4{ }^{\circ} \mathrm{C}$. Next, the cell pellet was washed in Dulbecco's phosphate-buffered saline (DPBS) and then pelleted using centrifugation. The cells were then treated with a solution of $1 \mu \mathrm{M}$ calcien AM and $2 \mu \mathrm{M}$ ethidium homodimer in DPBS for $20 \mathrm{~min}$ at room temperature. The cells were rinsed three times before suspending them in the core solution, which contained $2 \mu \mathrm{M}$ ethidium homodimer to track changes in cell viability within the particles after encapsulation.

4.7. Statistical Analysis. Analysis of variance (ANOVA) was used to identify both the main and the interaction effects significant in each response. This analysis was conducted at $95 \%$ confidence level (5\% significance). Insignificant main and interaction parameters were systematically eliminated from the model and a contour plot of each refined model was constructed. ANOVA calculations were all performed using Minitab statistical package (State College, PA).

\section{ASSOCIATED CONTENT}

\section{Supporting Information}

The Supporting Information is available free of charge on the ACS Publications website at DOI: 10.1021/acsomega.7b00570. Model diagnostic plots (PDF)

\section{AUTHOR INFORMATION}

\section{Corresponding Author}

*E-mail: lahann@umich.edu.

ORCID

Joerg Lahann: 0000-0002-3334-2053

Notes

The authors declare no competing financial interest.

\section{ACKNOWLEDGMENTS}

The authors would like to acknowledge funding from the Defense Threat Reduction Agency (HDTRA-1-15-1-0045).

\section{REFERENCES}

(1) Orive, G.; Santos, E.; Pedraz, J. L.; Hernandez, R. M. Application of cell encapsulation for controlled delivery of biological therapeutics. Adv. Drug Delivery Rev. 2014, 67-68, 3-14.

(2) Sun, A. M. Microencapsulation of Cells. Ann. N. Y. Acad. Sci. 1997, 831, 271-9.

(3) Sun, A. M.; O'Shea, G. M. Microencapsulation of living cells - A long-term delivery system. J. Controlled Release 1985, 2, 137-141.

(4) Murua, A.; Portero, A.; Orive, G.; Hernandez, R. M.; de Castro, M.; Pedraz, J. L. Cell microencapsulation technology: towards clinical application. J. Controlled Release 2008, 132, 76-83.

(5) Orive, G.; Hernandez, R. M.; Gascon, A. R.; Calafiore, R.; Chang, T. M. S.; de Vos, P.; Hortelano, G.; Hunkeler, D.; Lacik, I.; Pedraz, J. L. History, challenges and perspectives of cell microencapsulation. Trends Biotechnol. 2004, 22, 87-92.

(6) Wan, J. D. Microfluidic-Based Synthesis of Hydrogel Particles for Cell Microencapsulation and Cell-Based Drug Delivery. Polymers 2012, 4, 1084-1108.

(7) Gasperini, L.; Mano, J. F.; Reis, R. L. Natural polymers for the microencapsulation of cells. J. R. Soc., Interface 2014, 11, No. 20140817.

(8) Qiu, Y.; Park, K. Environment-sensitive hydrogels for drug delivery. Adv. Drug Delivery Rev. 2012, 64, 49-60.

(9) Provenzano, P. P.; Inman, D. R.; Eliceiri, K. W.; Knittel, J. G.; Yan, L.; Rueden, C. T.; White, J. G.; Keely, P. J. Collagen density promotes mammary tumor initiation and progression. BMC Med. 2008, 6, 11 
(10) Yang, S. H.; Ko, E. H.; Choi, I. S. Cytocompatible Encapsulation of Individual Chlorella Cells within Titanium Dioxide Shells by a Designed Catalytic Peptide. Langmuir 2012, 28, 2151-2155.

(11) Park, J. H.; Kim, K.; Lee, J.; Choi, J. Y.; Hong, D.; Yang, S. H.; Caruso, F.; Lee, Y.; Choi, I. S. A cytoprotective and degradable metalpolyphenol nanoshell for single-cell encapsulation. Angew. Chem., Int. Ed. 2014, 53, 12420.

(12) Zhang, W.; He, X. M. Encapsulation of living cells in small (approximately 100 micron) alginate microcapsules by electrostatic spraying: a parametric study. J. Biomech. Eng. 2009, 131, No. 074515. (13) Kobayashi, T.; Aomatsu, Y.; Iwata, H.; Kin, T.; Kanehiro, H.; Hisanaga, M.; Ko, S.; Nagao, M.; Nakajima, Y. Indefinite islet protection from autoimmune destruction in nonobese diabetic mice by agarose microencapsulation without immunosuppression. Transplantation 2003, 75, 619-625.

(14) Sugiura, S.; Oda, T.; Izumida, Y.; Aoyagi, Y.; Satake, M.; Ochiai, A.; Ohkohchi, N.; Nakajima, M. Size control of calcium alginate beads containing living cells using micro-nozzle array. Biomaterials 2005, 26, 3327-3331.

(15) Khademhosseini, A.; May, M. H.; Sefton, M. V. Conformal coating of mammalian cells immobilized onto magnetically driven beads. Tissue Eng. 2005, 11, 1797-1806.

(16) Murua, A.; de Castro, M.; Orive, G.; Hernandez, R. M.; Pedraz, J. L. In vitro characterization and in vivo functionality of erythropoietin-secreting cells immobilized in alginate-poly-L-lysinealginate microcapsules. Biomacromolecules 2007, 8, 3302-3307.

(17) Roh, K. H.; Martin, D. C.; Lahann, J. Biphasic Janus particles with nanoscale anisotropy. Nat. Mater. 2005, 4, 759-763.

(18) Roh, K. H.; Martin, D. C.; Lahann, J. Triphasic nanocolloids. J. Am. Chem. Soc. 2006, 128, 6796-6797.

(19) Roh, K. H.; Yoshida, M.; Lahann, J. Water-stable biphasic nanocolloids with potential use as anisotropic imaging probes. Langmuir 2007, 23, 5683-5688.

(20) Yoshida, M.; Roh, K. H.; Lahann, J. Short-term biocompatibility of biphasic nanocolloids with potential use as anisotropic imaging probes. Biomaterials 2007, 28, 2446-2456.

(21) Yarin, A. L.; Koombhongse, S.; Reneker, D. H. Bending instability in electrospinning of nanofibers. J. Appl. Phys. 2001, 90, $4836-4846$.

(22) Kazemi, A.; Lahann, J. Environmentally responsive core/shell particles via electrohydrodynamic co-jetting of fully miscible polymer solutions. Small 2008, 4, 1756-1762.

(23) Hwang, S.; Roh, K. H.; Lim, D. W.; Wang, G. Y.; Uher, C.; Lahann, J. Anisotropic hybrid particles based on electrohydrodynamic co-jetting of nanoparticle suspensions. Phys. Chem. Chem. Phys. 2010, 12, 11894-11899.

(24) Chakraborty, S.; Liao, I. C.; Adler, A.; Leong, K. W. Electrohydrodynamics: A facile technique to fabricate drug delivery systems. Adv. Drug Delivery Rev. 2009, 61, 1043-1054.

(25) Gasperini, L.; Maniglio, D.; Migliaresi, C. Microencapsulation of cells in alginate through an electrohydrodynamic process. J. Bioact. Compat. Polym. 2013, 28, 413-425.

(26) Box, G. E. P.; Hunter, W. G.; Hunter, J. S. Statistics for Experimenters: An Introduction To Design, Data Analysis, and Model Building; Wiley: New York, 1978; p viii.

(27) Bhaskar, S.; Pollock, K. M.; Yoshida, M.; Lahann, J. Towards designer microparticles: simultaneous control of anisotropy, shape, and size. Small 2010, 6, 404-411.

(28) Hartman, R. P. A.; Brunner, D. J.; Camelot, D. M. A.; Marijnissen, J. C. M.; Scarlett, B. Jet break-up in electrohydrodynamic atomization in the cone-jet mode. J. Aerosol Sci. 2000, 31, 65-95.

(29) Lahann, J. Recent progress in nano-biotechnology: compartmentalized micro- and nanoparticles via electrohydrodynamic cojetting. Small 2011, 7, 1149-1156.

(30) Champion, J. A.; Walker, A.; Mitragortri, S. Role of particle size in phagocytosis of polymeric microspheres. Pharm. Res. 2008, 25, $1815-1821$.
(31) Yang, Y.; Bajaj, N.; Xu, P.; Ohn, K.; Tsifansky, M. D.; Yeo, Y. Development of highly porous large PLGA microparticles for pulmonary drug delivery. Biomaterials 2009, 30, 1947-1953.

(32) Impellitteri, N. A.; Toepke, M. W.; Levengood, S. K. L.; Murphy, W. L. Specific VEGF sequestering and release using peptidefunctionalized hydrogel microspheres. Biomaterials 2012, 33, 34753484.

(33) Duncanson, W. J.; Figa, M. A.; Hallock, K.; Zalipsky, S.; Hamilton, J. A.; Wong, J. Y. Targeted binding of PLA microparticles with lipid-PEG-tethered ligands. Biomaterials 2007, 28, 4991-4999. 